\title{
Recherche sur les parasites internes des Éphéméroptères de la région de Cracovie
}

\author{
par Lucie ARVY * et R. SOWA ** \\ * Laboratoire d'Histo-enzymologie, Faculté de Médecine, 45, rue des Saints-Pères, \\ F 75006 Paris \\ ** Instytut Zoologii, Universytet Jagiellonski, Cracovie, Pologne
}

\section{Résumé.}

Pour la première fois, sept sortes de parasites internes: Spiriopsis adipophila, Telomyxa glugeiformis, métacercaire de Crepidostomum farionis, Cystidicoloides sp. et des Microsporidies, Nématomorphe et Gordiacé indéterminés, sont signalés chez des Ephéméroptères de Pologne.

\section{Summary.}

Researches on the internal parasites in Ephemeroptera around Cracovie.

For the first time, 7 kinds of internal parasites/Spiriopsis adipophila, Telomyxa glugetformis, cysts of Crepidostomum farionis, Cystidicoloides and undetermined species of Microsporidia, Nematomorpha and Gordiacea are encountered in polish Ephemeroptera.

\section{Matériel}

Il ne semble pas que quelque parasitisme ait été jamais suspecté chez les larves éphémériennes polonaises; c'est une des raisons qui motivent la publication de nos observations toutes préliminaires.

Nous avons pu examiner (tableau $I$ ) des représentants de 5 espèces appartenant à 4 genres d'Ephéméroptères ; 22 larves ont été récoltées dans la rivière Pradnik-Bialucha: Baetis rhodani (Pictet), Ephemera danica O. F. Müller, Ephemerella ignita (Poda) 
TABLEAU I. - Le parasitisme chez les Ephéméroptères polonais.

\begin{tabular}{|c|c|c|c|c|c|c|c|c|}
\hline Origine & $\begin{array}{l}\text { Ephéméroptères } \\
\text { Espèces et nombres }\end{array}$ & S & $\mathrm{T}$ & M & $\mathrm{N}$ & $\mathrm{Gr}$ & K & Go \\
\hline \multirow{3}{*}{$\begin{array}{l}\text { Pradnik- } \\
\text { Bialucha }\end{array}$} & Baetis rhodani (Pict.) & 0 & 0 & 2 & 0 & 0 & 0 & 0 \\
\hline & $\begin{array}{c}\text { Ephemera danica O.F. Müll. } \\
17\end{array}$ & 17 & 17 & 0 & 12 & 17 & 1 & 0 \\
\hline & $\underset{3}{\text { Ephemerella ignita (Poda) }}$ & 3 & 0 & 0 & 0 & 0 & 0 & 0 \\
\hline \multirow{5}{*}{ Sanka } & Baetis muticus (L.) & 0 & 0 & 0 & 0 & 0 & 0 & 3 \\
\hline & Baetis rhodani (Pict.) & 0 & 0 & 0 & 0 & 1 & 0 & 0 \\
\hline & $\begin{array}{c}\text { Ecdyonurus starmachi Sowa } \\
9\end{array}$ & 0 & 0 & 0 & 0 & 0 & 0 & 0 \\
\hline & Ephemera danica O.F. Müll. & 2 & 2 & 0 & 2 & 0 & 0 & 0 \\
\hline & $\underset{6}{\text { Ephemerella ignita (Poda) }}$ & 4 & 0 & 0 & 0 & 0 & 0 & 0 \\
\hline
\end{tabular}

$\mathrm{S}=$ Spiriopsis adıpophila (Arvy et Delage); $\mathrm{T}=$ Telomyxa glugeiformis Léger et Hesse.

$\mathrm{M}=$ Microsporidies $; \quad \mathrm{N}=$ Nématodes; $\mathrm{Gr}=$ Grégarines; $\mathrm{K}=$ kystes métacercariens ; Go = larves de Gordiacé.

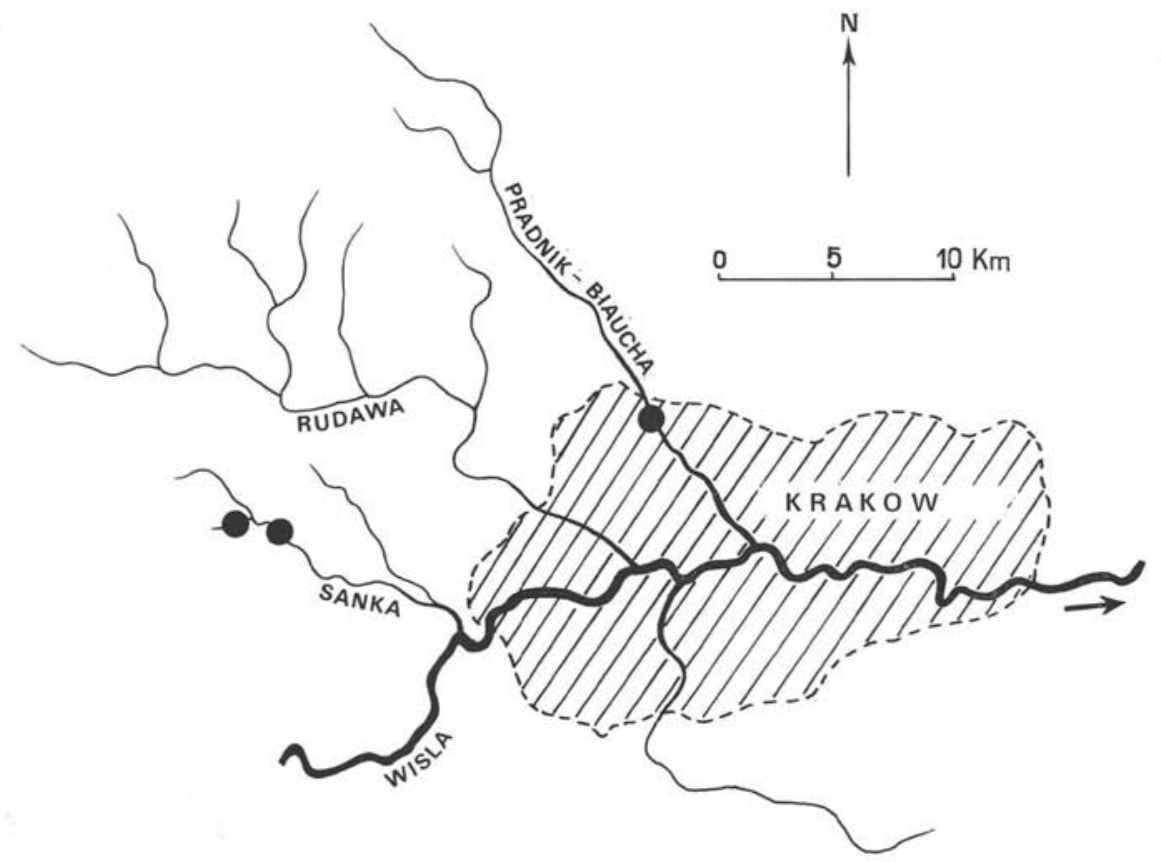

CARTE 1. - Situation des trois gîtes d'éphéméroptères explorés, au nord-ouest de Cracovie. 
et dans la Sanka, ruisseau de la vallée Dolina Mnikowska, nous avons trouvé les mêmes espèces avec, en outre, des Ecdyonurus starmachi Sowa et des Baetis muticus (L.), (soit 27 larves). Ces larves ont été récoltées fin août, en trois points (carte 1) bien souvent explorés par l'un de nous (R.S.).

\section{Observations}

Les larves d'E. starmachi sont remarquablement peu parasitées: nous n'avons: trouvé que de rares larvules d'un Nématomorphe indéterminé chez ces éphémères, récoltées près de l'origine de la Sanka, dans un ruisselet aux eaux vives et à fond caillouteux.

Les larves de Baetis muticus sont également très saines; elles seraient indemnes. de parasite visible à l'œil nu, ou avec une bonne loupe, si elles ne contenaient pas, très fréquemment (dans plus de la moitié des cas), des kystes d'un Gordiacé (fig. 1), proche du Neochordodes occidentalis (Montgomery, 1898), étudié récemment par Poinar et Doelman (1974); ces chercheurs ont pu infester expérimentalement des éphémères non identifiées avec ce nématomorphe.

Les Baetis rhodani de la Pranik-Bialucha contenaient des microsporidies non déterminées et l'une des larves de la Sanka était remarquablement riche en grégarines (fig. 2).

Les Ephemerella ignita sont assez riches en Spiriopsis adipophila, quel que soit leur lieu d'origine (fig. 3), mais l'infestation est moins fréquente chez les éphémères de la Sanka.

Les 17 larves d'Ephemera danica examinées contenaient de très nombreux Spiriopsis adipophila (fig. 4) et d'encore plus nombreuses microsporidies, semblables à celles décrites par Léger et Hesse, à la fin du siècle dernier, sous le nom de Telomyxa glugeiformis et étudiées plus récemment en 1961 (Codreanu) et 1970 (Codreanu et Vavra) ; en outre, les larves contenaient, dans plus de la moitié des cas, des larvules de toutes tailles identifiées par le $\mathrm{P}^{r}$ Chabaud et $\mathrm{M}^{\mathrm{me}}$ Petter comme des Cystidicoloides; l'une des larves portait de nombreux kystes métacercariens, pyriformes, contenant une ophthalmocercaire pourvue d'un stylet: Crepidostomum farionis O.F. Müller.

EN CONCLUSION : les larves polonaises de Baetis (rhodani et muticus) et d'Ecdyonurus starmachi sont très saines, mais les larves d'Ephemera danica sont si intensément parasitées que l'espèce semble être appelée à disparaître, dans les gîtes explorés ; en effet, aucune des larves examinées ne contenaient de gonade différenciée, le parasitisme réalisant une véritable castration.

Les résultats de la brève étude parasitologique à laquelle nous nous sommes livrés prêtent à des discussions variées; les unes se limitent aux espèces que nous avons étudiées, les autres ont une portée plus générale, avec des incidences sur les cycles vitaux, les particularités des émergences d'une zone géographique à une autre et les analyses phylétiques intercontinentales. 

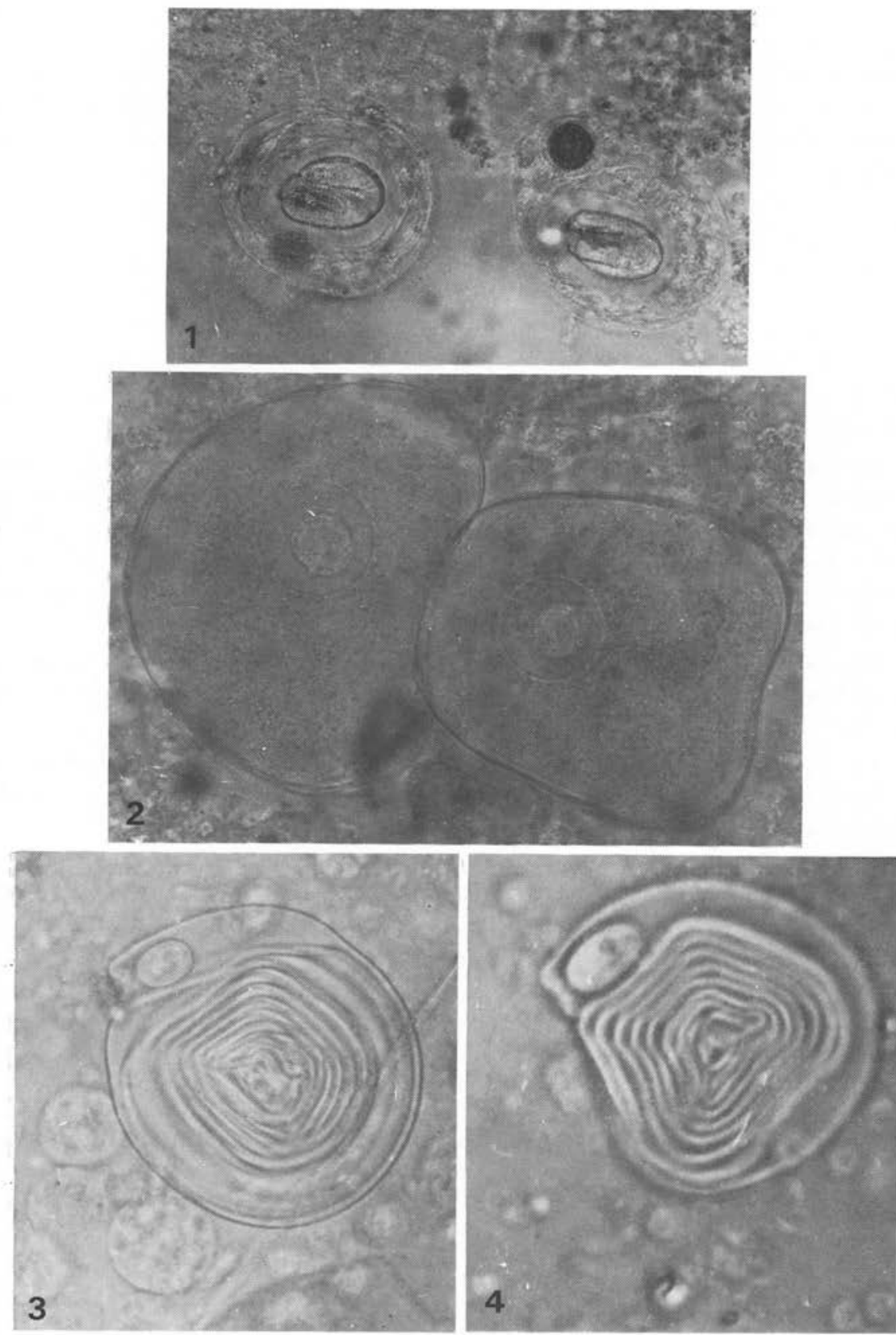

FIG. 1 à 4. - Aspect des larves de Gordiacé enkystées chez les Baetis muticus (L.) de la Sanka. FIg. 2. - Aspect d'un type de syzygie chez les Grégarines de Baetis rhodani (Pict). FIG. 3. - Spiriopsis aäipophila (Arvy et Delage, 1966) chez Ephemera danica O.F. Müller. FIG. 4. - Le même parasite chez Ephemerella ignita (Poda). 


\section{$I^{\circ}$ Remarques quant aux espèces examinées.}

Si les parasites que nous avons décelés étaient inconnus en Pologne, aucun d'eux n'est cependant nouveau pour la science.

a) Spiriopsis adipophila est actuellement connu (carte 2) chez Ephemera vulgata (France et Grand-Duché de Luxembourg), Ephemera danica (Grand-Duché de Luxembourg et Pologne), Ephemerella ignita (Grand-Duché de Luxembourg et Pologne), Baetis bioculatus et B. rhodani, Centroptilum luteolum, Chitinophora krieghoffi, Cloeon dipterum et C. inscriptum, Potamanthus luteus, Torleya belgica (Grand-Duché de Luxembourg) (1).

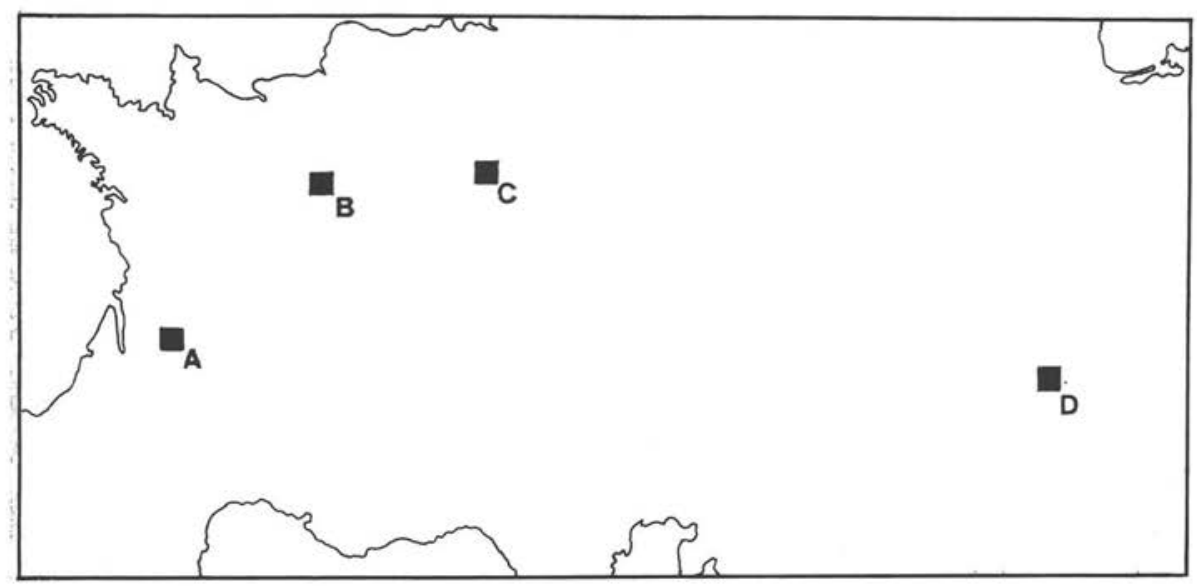

CARTE 2. - Les zones infestées par Spiriopsis adipophila: A : région des Eyzies (Arvy et Delage); B: Bassin parisien: Avre (Chabaud et Arvy); Le Cléry (Arvy et Domergue) ; C: Grand Duché de Luxembourg (Hoffmann et Delvaux); D: région de Cracovie (Arvy et Sowa).

b) Telomyxa glugeiformis, microsporidie à spores ellipsoïdes $(6,5 \times 4 \mu)$, réfringentes et pourvue de deux capsules polaires, découverte chez les Ephemera vulgata de France par Léger et Hesse (1910) a été retrouvée chez les Ephemera danica de Roumanie (Codreanu, 1961) et de Bordeaux (Codreanu et Vavra, 1970). Cependant, l'infestation des Ephemera danica est si massive chez les larves polonaises que toute trace de tissu adipeux et de gonade a disparu, sans qu'il soit possible de préciser les parts respectives des Spiriopsis adipophila et des Telomyxa glugeiformis dans cette disparition, tant ces deux parasites spoliateurs sont abondants.

(1) Dans une lettre récente, le $\mathrm{P}^{r}$ W.-L. Peters a la bonté de nous informer que, par Acte de la Commission de nomenclature, Baetis bioculatus est devenu Baetis fuscatus, Chitonophora krieghoffi est devenu Ephemerella krieghoffi et Torleya belgica est devenu synonyme de Torleya major. 
c) L'infestation des éphémères par des kystes métacercariens de Crepidostomum farionis a été découverte en Angleterre par Brown (1927) et signalée au Canada par Choquette (1954); si elle peut être importante, elle n'est pas fréquente, chez les éphémères des deux rivières polonaises.

d) Des larvules identiques à celles que nous avons vues chez Ephemera danica avaient été signalées chez Hexagenia recurvata Morgan et chez Polymitarcys sp. au Canada (Choquette, 1955); Moravec (1971) a réalisé l'infestation expérimentale de larves d'Habrophlebia lauta, Habroleptoides modesta et d'Ephemera danica, mais une telle infestation naturelle était encore inconnue en Europe. Comme dans les infestations expérimentales de Moravec, nos larvules de Cystidicoloides étaient très actives dans le milieu intérieur des larves d'Ephemera danica; aucune d'elles ne manifestait de tendance à l'enkystement et leurs tailles étaient très variées ; cependant, aucune n'était adulte; Moravec a d'ailleurs montré que les larves parasites croissent et muent chez l'éphémère, mais ne peuvent achever leur cycle vital et pondre que chez leur hôte définitif : un Salmonidé.

e) On sait que des larves de Gordiidae peuvent parasiter et évoluer dans la cavité générale de certains Insectes (Coléoptères, Orthoptéroïdes, Chironomides, Trichoptères, Odonates), de certains Myriapodes, voire d'Arachnides (Dorier, 1965) ; mais chez les éphémères, les larves de Gordiidae n'évoluent pas : peu après leur pénétration, elles s'enkystent, les éphémères ne jouant qu'un rôle de transporteur, intermédiaire pour l'hôte définitif. On sait néanmoins, depuis environ 120 ans, que les larves de Gordiidae peuvent trouver abri et persister chez les larves d'éphémères: Meissner (1856) après avoir mis en contact des larves de Gordius aquaticus et des larves d'éphémères, retrouva des kystes dans tous les organes et jusque dans les tarses, les tibias, les fémurs, les muscles (fig. 5 à 10); depuis ces temps lointains, des larves de Gordiidae sont signalées par intermittences, chez Cloeon dipterum, il s'agissait suivant Linstow (1891-1892) de Parachordodes tolosanus, étudié récemment par Dorier (1965); les Leptophlebia et Ephemerella peuvent être parasitées par Paragordius varius (White, 1966-1969) ; suivant Inoué (1960-1962), les Cloeon dipterum du Japon peuvent héberger des Chordodes japonensis; enfin, en Californie, Poinar et Doelman ont pu infester expérimentalement des larves d'éphémères non identifiées avec des larves de Neochordodes occidentalis. A la lumière de nos observations, l'un de nous (L. A.), reprenant l'examen de coupes histologiques de divers éphéméroptères, faites (en 1952) en vue de recherches d'un autre ordre, a reconnu des kystes d'un Gordiidae chez une larve d'Ecdyonurus venosus, récoltée dans un affluent de la Vienne, en Limousin; l'éphémère ne contenait pas moins de 7 kystes. L'identification des larves enkystées est particulièrement difficile. On sait que les Neochordodes et les Pseudochordodes sont limités à l'Amérique, les Paragordidae sont connus d'Afrique et d'Amérique du Sud, les Parachordodidae sont africains et des Euchordodes ont été signalées en Europe. Des Chordodes sont connus chez certains Insectes (Blatta, Forficula, Mantis, Periplaneta), et chez certains Arachnides (Phriniscus bacillifer). Il ne semble pas qu'il y ait d'hôtes spécifiques pour les larves de Gordiacés et cependant Montgomery (1898) a admis que certains Coléoptères s'infestent en dévorant des larves d'éphémères. 

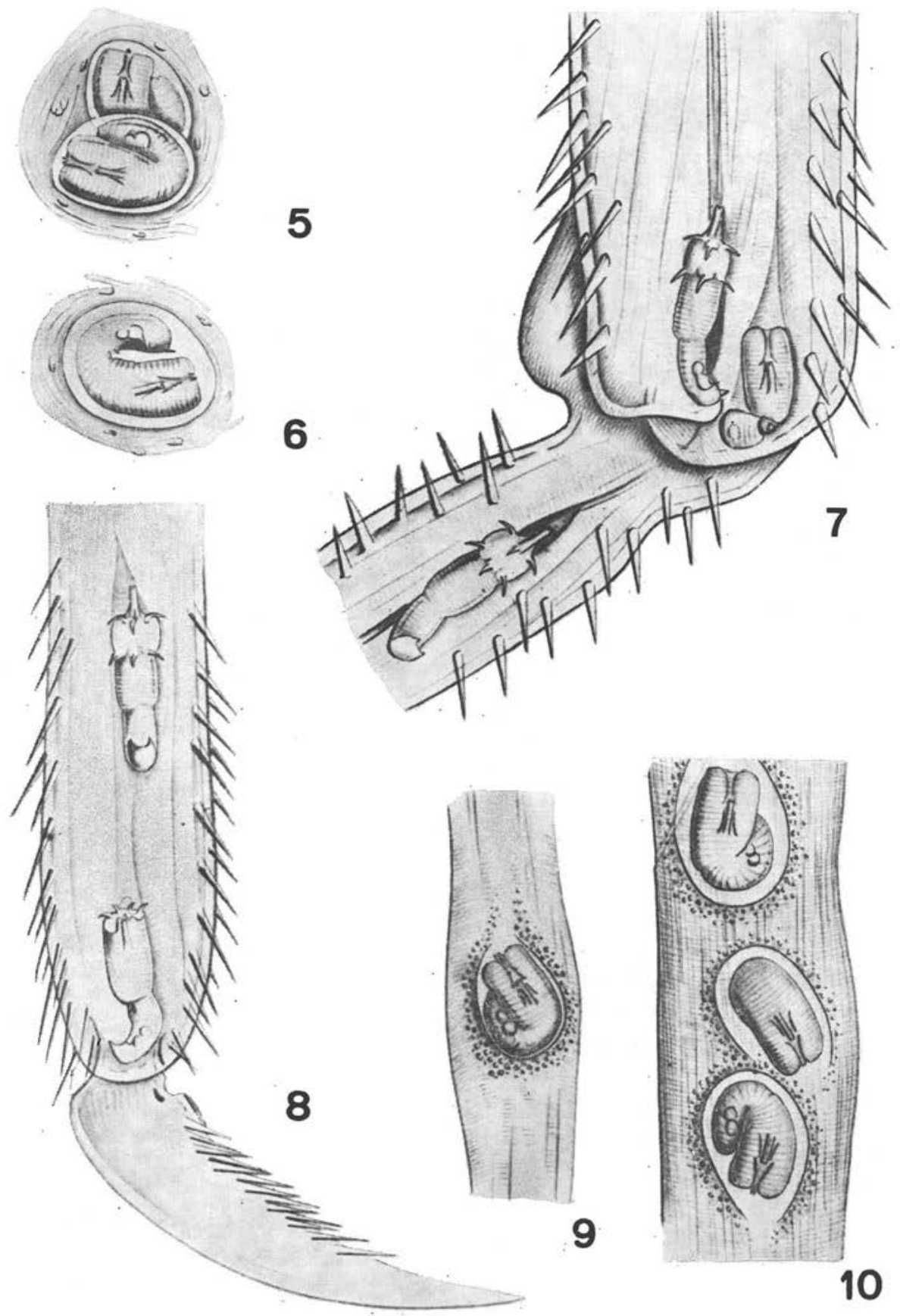

FIG. 5 à 10. - Aspect des larves de Gordius aquaticus, après infestation expérimentale de larves d'éphémères (d'après Meissner, 1856). 5-6 - les kystes ne contiennent très généralement qu'une seule larve, mais parfois ils en contiennent deux. 7-8 - larves en migration au niveau des: fémur, tibia et tarse. 9-10 — larves en voie d'enkystement entre des fibres musculaires. 


\section{$2^{\circ}$ Remarques générales.}

A l'heure actuelle, nul chercheur n'a envisagé quelque influence des divers parasitismes sur les cycles vitaux chez les éphéméroptères; or, si la durée de chaque stade larvaire est liée au type d'alimentation des larves (Cianciara, 1975), il n'est pas invraisemblable d'admettre que des parasitismes massifs, par Spiriopsis adipophila, ou par Telomyxa glugeiformis, agents spoliants, provoquant la disparition du tissu adipeux, puisse modifier le nombre ou la durée des stades larvaires.

De même, pour expliquer les différences entre les émergences des éphéméroptères observées d'une année à l'autre, ou d'une région à l'autre, seules des fluctuations de la température, de la force des vents, de l'abondance ou de la rareté des pluies..., etc..., ont été retenues; il n'est pas invraisemblable d'admettre qu'un parasitisme intercurrent puisse influencer, hâter ou retarder les émergences.

Enfin, il n'est pas parfaitement rationnel de comparer, par exemple, des populations en Europe centrale et en Scandinavie, en rapportant exclusivement leurs différences à la seule altitude, à la seule température moyenne, à la force des courants, aux. différences de végétation du lit, ou à la nature du fond ; il pourrait être bon de s'assurer de l'absence, sinon de la plus ou moins grande abondance de divers parasites dans. les régions géographiques comparées. De même, il est vraisemblable que les analyses phylétiques et les hypothétiques échanges de faune entre éphéméroptères d'un continent. à l'autre, pourraient trouver quelque argument dans les identités, ou les différences, de leurs parasites. En conclusion, dans l'absolu, il n'est pas exact de prétendre connaître les cycles évolutifs des éphéméroptères ou la dynamique des populations d'éphéméroptères si on ignore leurs parasites.

\section{Bibliographie}

ARVY (L.), 1952. - Présence de larves enkystées d'un Gordiacé chez une larve âgée d'Ecdyomurus venosus. Fab. (inédit).

ARVy (L.) et Delage (B.), 1970. - Permanence de l'infestation des Ephemera vulgata desEyzies par Spirinella adipophila Arvy et Delage, 1966. First Int. Conf. on Ephemeroptera, Tallahassee, 215-222, 3 Pl. h.-t., 1 carte.

ARVY (L.), 1971. - Spirinella adipophila Arvy et Delage 1966, parasite d'Ephemera vulgata, une énigme. $1^{\text {er }}$ Multicolloque européen de Parasit., Rennes, 431.

ARVY (L.) et Peters (W. L.), 1973. - A new name for a generic homonym of a parasite in Ephemeroptera (Protozoa). Ent. News., 1972, 83, 38. - Phorésies, biocoenoses et thanatocoenoses chez les Ephéméroptères. Proc. 1st Int. Conf. Ephemeroptera. Brill, édit., Leiden, 254-312. — Phorésies, biocoenoses et thanatocoenoses in the Ephemeroptera. Supplement. Proc. 2nd Int. Conf. Ephemeroptera, Cracovie (àparaître).

ARVY (L.), 1975. - Nouvelles données sur Spiriopsis adipophila (Arvy et Delage, 1966), Sporozoaire probable. 2nd Int. Conf. on Ephemeroptera, Cracovie (à paraître). 
Chabaud (A.), 1976. - CIH keys to the Nematode parasites of Vertebrates. $\mathrm{N}^{\circ} 4$. Keys to genera of the Spiruroidea, Habronematoidea and Acuarioidea, in: ANDERSON, Chaвaud and Willmotr, Commonvealth Agricultura! Bureau, Farnham Royal, Bucks, England (à paraître).

Chopuette (L. P. E.), 1954. - A note on the intermediate hosts of the trematode Crepidostomum cooperi Hopkins, 1931, parasitic in speckled trout Salvenilus fontinalis (Mitchell) in some lakes and rivers of the Quebec Laurentide Park. Canad. J. Zool., 32, 375-7. - The life history of the nematode Metabronema salvelini (Fujita, 1920). parasitic in the speckled trout Salvelinus fontinalis (Mitchell) in Quebec. Canad. J. Zool., 1955, 33, 1-4.

Cianciara (S.), 1975. - Some study on biology and bioenergetics of Cloeon dipterum L. 2nd Int. Conf. on Ephemeroptera, Cracovie (à paraître).

Codreanu (R.), 1961. - Sur la structure bicellulaire des spores de Telomyxa cf. glugeiformis Léger et Hesse, 1910, pa-asite des nymphes d'Ephemera (France, Roumanie) et les nouveaux sous-ordres de Microsporidies Monocytosporea nov. et Polycytosporea nov. C.R. Acad. Sci., 253, 1613-5.

Codreanu (R.) et VAVRa (J.), 1970. - The structure and ultrastructure of the Microsporidan Telomyxa glugeiformis Léger and Hesse, 1910, parasite of Ephemera danica (Müll)nymphs. J. of Protozool., 17, 374-84.

Delvaux (L.). - Spiriopsis adipophila (Arvy et Delage, 1966), parasite constant des populations d'Epinemera danica (O. F. Mull.) du Grand-Duché de Luxembourg. Proc. 2nd. Conf. Ephemeroptera, Cracovie (à paraître).

Dorier (A.), 1930. - Recherches biologiques et systématiques sur les Gordiacés. Trav. Lab. Hydrob. Pisc. Grenoble, 22, 1-184. - Classe des Gordiacés v. Siebold 1943. (= Nematomorpha Vejdovsky 1886). In P. P. Grassé, Traité de Zoologie, 4, 12011222, Masson, édit., Paris.

INOUE (I.), 1960. - Studies on the life history of Chordodes japonensis a species of Gordiacea. Annot. Zool., Japon., 33, 132-41 et 35, 12-19.

Léger (L.) et Hesse (E.), 1910. - Cnidosporidies des larves d'éphémères. C.R. Acad. Sci., 150, 411-4. - Microsporidies bactériformes et essai de systématique du groupe. C.R. Acad. Sci., 1922, 174, 327-30.

Linstow (O. F. B. von), 1891-1892. - Weitere Beobachtungen an Gordius tolosanus und Mermis. Arch. mikr. Ancit., 37, 239-49. - Beobachtungen an Helminthenlarva. Arch. mikr. Anat., 39, 325-42.

Meissner (G.), 1856. - Beiträge zur Anatomie und Physiologie der Gordiaceen. Zeit. wiss. Zool., 7, 1-140.

Maggenti (A. R.) et Paxman (G. A.), 1971. - Sterliodochona pedispicula sp., n. (Nematoda: Spirurinae) from Salmo gairdnerii Richardson, and a discussion of the genera Sterliodochona Skrjabin 1936 and Cystidicoloides Skinker, 1931. Proc. Helminthol. Soc. Wash., 38, 210-4.

Montgomery (T. H.), 1898. - The Gordiacea of certain american collections... Bull. Mus.. comp. Zool., 32, 1-59 et Proc. California Acad. Sci., 1, 333-44. 
Moravec (F.), 1971. - On the life history of the nematode Cystidicoloides tenuissima (Zeder, 1800 ) in the river Bystrice, Czechoslovakia. Folia Parasitol., 1971, 18, 107-12. -Studies ont the development of the nematode Cystidicoloides tenuissima (Zeder, 1800). Vest. Cesk. Spol. Zool., 35, 43-55.

Poinar (G. O.) et Doelman (J. J.), 1974. - A reexamination of Neochordodes occidentalis (Montg.) comb. n. (Chordodidae: Gordioidea): larval penetration and defense reaction in Culex pipiens L. J. of Parasitol., 60, 327-35.

Sowa (R.), 1971. - Ecdyonurus starmachi sp. n. et E. submontanus Landa des Carpates polonaises (Ephemeroptera: Heptageniidae). Bull. Acad. pol. Sci., Sér. II. Sc. biol,, 19, 407-12.

White (D. A.), 1960. - A new host record for Paragordius varius (Nematomorpha). Trans. amer. micr. Soc., 1966, 85, 579. - The infection of immature aquatic insect by larval Paragordius (Nematomorpha). Great Basin Natur., 29, 44. 